\title{
Educación en niños asmáticos controlados en hospital Roberto del Río. Impacto en conocimientos, adherencia y control de la enfermedad
}

\author{
MARÍA TERESITA HENRÍQUEZ Y.*** y ELIANA CERUTI D.**
}

Education in asthmatic children controlled at Roberto del Rio Hospital. Impact on knowledge, adhesion and disease control

Introduction: Today is clear the role of educational programs in the control of chronic diseases. The goal was optimize the knowledge, adhesion, inhalation technique and asthma control through an educational workshop. Methods: We enrolled asthmatic patients (age 5 to 14 years), with questionnaire "control of asthma in children" (CAN). Patients with an score greater than or equal to 8 were invited to the educational workshop with a class of asthma, after a evaluation of knowledge and inhalation technique. These patients were reevaluated 6 months later. Results: 91 patients were recruited with a CAN score greater than or equal to 8, only 49 attended the workshop and 40 attended the reevaluation. Of these, 52,5\% of the patients achieved a CAN score less than 8 . Two patients were excluded because of modification of treatment, resulting in 47,5\% of patients with CAN score less than 8 . Conclusion: The education improved the knowledge, inhalation technique and adherence. Educational workshop should be implemented in our clinical practice.

Key words: Asthma education, pediatric asthma.

\section{Resumen}

Introducción: El objetivo fue optimizar los conocimientos, técnica inhalatoria, adherencia y el control del asma, mediante un taller de educación. Métodos: Se enrolaron pacientes asmáticos (edad: entre 5 y 14 años), con cuestionario CAN (Control del Asma en Niños). Los pacientes con puntaje mayor o igual a 8, se invitaron a un taller de educación, se evaluó conocimientos, técnica inhalatoria y se realizó una clase sobre asma. A los 6 meses, se reevaluaron con cuestionario CAN, prueba de conocimientos y técnica inhalatoria. Resultados: Se reclutaron 91 pacientes con CAN mayor o igual a 8, de los cuales asistieron 49 al taller. En la segunda etapa asistieron 40 pacientes. En el 52,5\% de los pacientes, se logró puntaje CAN menor a 8 puntos. Se excluyeron 2 pacientes, por modificación del tratamiento, como resultado final 47,5\% de los pacientes, disminuyó su puntaje CAN a menos de 8. Conclusiones: La educación mejoró conocimientos, técnica inhalatoria y adherencia. Se deberían implementar talleres educativos en nuestra práctica clínica.

Palabras clave: Educación en asma, asma pediátrica.

\section{Introducción}

En la actualidad resulta indiscutible el papel que la educación juega en el manejo de las enfermedades crónicas, y el asma no es una excepción. A pesar de que la educación, es reconocida como uno de los pilares del tratamiento, la literatura ha demostrado conclusiones divergentes ${ }^{1}$. Así al revisar la última publicación Cochrane, sobre aplicación de programas de educación en poblaciones con asma, la conclusión es que aún los datos son limitados y que la educación mejoraría sólo algunos aspectos de la enfermedad, como calidad de vida, conocimientos en asma y reducción de

* Becada de Enfermedades Respiratorias Pediátricas.

** Facultad de Medicina, Universidad de Chile, Hospital Roberto del Río. 
exacerbaciones $^{2}$. Otros autores han demostrado reducción en el número de hospitalizaciones y visitas a urgencia ${ }^{3}$.

La educación en asma ayudaría a los padres y niños, a entender su enfermedad, y en teoría el aumento de conocimientos y mejor adherencia al tratamiento, debería ir ligado a un mejor control del asma ${ }^{4}$. No existe una forma única de educar, que asegure el logro de todos nuestros objetivos, sin embargo, se encuentran bien definidos conceptos, que deben ser entregados en todo programa de educación, como desencadenantes de crisis, explicación básica de la enfermedad e instrucción de la técnica inhalatoria ${ }^{5}$.

El objetivo principal de este trabajo, fue mejorar el control del asma, evaluado según cuestionario CAN (Control del Asma en Niños). La intervención realizada fue un taller de educación, realizado a los pacientes y sus cuidadores. El cuestionario CAN es una herramienta diseñada para evaluar el control del asma en niños, desarrollada y validada en población española, posee dos versiones, para tutores ( 2 a 8 años) y niños/ adolescentes (9 a 14 años) ${ }^{6}$. Se ha establecido que obtener un puntaje CAN mayor o igual a 8 puntos, es indicador de mal control ${ }^{7}$, así a los pacientes que obtuvieron un puntaje mayor o igual a 8 , se les invitó a asistir a este taller de educación en asma bronquial.

Nuestros objetivos secundarios fueron optimizar los conocimientos básicos sobre asma bronquial en los cuidadores, evaluado a través de una prueba de conocimientos, optimizar la técnica inhalatoria y mejorar la adherencia.

\section{Material y Método}

Se enrolaron pacientes, con edad entre 5 y 14 años, controlados en el policlínico de Broncopulmonar del Hospital Roberto del Río, con diagnóstico de Asma Bronquial, durante el período comprendido entre julio y diciembre del año 2009. Los pacientes eran enrolados, al asistir al laboratorio de función pulmonar. Se les aplicó el cuestionario CAN (Control del Asma en Niños). Este cuestionario fue realizado a los cuidadores (versión tutores) en el caso de los pacientes menores de 9 años y fue realizado a los pacientes (versión niños/adolescentes), en el caso de pacientes con edad mayor o igual a 9 años. Se les solicitó datos de contacto (nombre del paciente y su cuidador, edad y teléfono).

A los pacientes que obtenían un puntaje en el cuestionario CAN, mayor o igual a 8 , indicador de mal control, se les invitó, a través de un llama- do telefónico, a asistir a un Taller de Educación en Asma bronquial, tanto al cuidador como al paciente. El taller se realizó en las dependencias del Hospital Roberto del Río.

Al inicio del taller de educación, los cuidadores completaban unas preguntas sobre el tratamiento actual indicado para el asma de su hijo, con nombre del medicamento, dosis, horario de administración, tratamiento adicional por otra patología y una última pregunta sobre adherencia, donde los cuidadores, debían contestar cuantos días a la semana (de 1 a 7), no administraban el medicamento, ya sea por olvido del cuidador o del paciente. Posteriormente los cuidadores realizaron una prueba de 14 preguntas, sobre conocimientos básicos de Asma Bronquial y tratamiento, basada en afirmaciones, a las cuales los cuidadores debían contestar "Sí" en caso de estar de acuerdo y "No" en caso de no estar de acuerdo. Los resultados fueron ponderados con una nota de 1 a 7 . Después de completar la prueba de conocimientos, se realizó una clase elaborada en power point, de aproximadamente $30 \mathrm{~min}$, con conceptos y dibujos, en modalidad interactiva, donde los cuidadores y pacientes, podían interrumpir y hacer preguntas.

Entre los contenidos entregados, destacó la información sobre definición de asma, patogenia básica de la enfermedad, gatillantes de crisis, reconocimiento de crisis de asma y su manejo inicial, además de conceptos sobre los tratamientos y las formas de administración. Durante el desarrollo del taller, se invitó a cuidadores y pacientes, a reproducir en forma práctica, la técnica inhalatoria para la administración del tratamiento del asma; para esta demostración se les entregó una aerocámara y un inhalador de dosis medida. Mientras demostraban la técnica, se evaluó mediante una pauta con nota, si se cumplían los pasos adecuados para una óptima técnica inhalatoria, con una nota entre 1 a 7. Posteriormente, se reforzó la técnica en caso de ser correcta, o se enseñó y corrigió lo realizado, a través de la demostración práctica y luego la reproducción en forma correcta, por parte de los niños y sus cuidadores, en forma repetida si era necesario. Al finalizar la clase, se les solicitó a los cuidadores y niños, una evaluación por escrito del taller realizado, con sus impresiones y críticas. Se les entregó un folleto para la casa, especialmente diseñado con este fin, con un resumen de los contenidos entregados y las indicaciones de manejo inicial en caso de una crisis de asma.

Tras 4 a 6 meses de realizado el taller de educación, se contactó a los pacientes nuevamente por teléfono y se les solicitó asistir a una segun- 
da sesión, para realizar una reevaluación, en la cual se aplicó el cuestionario CAN, se repitió la misma prueba de conocimientos, aplicada en la primera sesión, completaron el cuestionario sobre tratamiento y adherencia, respondiendo el tratamiento actual del asma, nuevos tratamientos adicionales y días de la semana que no fue administrado el tratamiento. Posteriormente se reevaluó la técnica inhalatoria, con nota según la pauta anterior, nuevamente se corrigió en caso de no ser óptima, mediante demostración práctica y repetición de la técnica por parte del paciente y su cuidador. Esta segunda sesión, se utilizó para reforzar conocimientos sobre asma y responder las dudas surgidas durante el período entre sesiones. Sobre los cálculos estadísticos se estimó un tamaño muestral de 24 pacientes, para demostrar una mejoría en el control del asma (CAN menor a 8 ) en el 30\% de los pacientes, con una estimación de error alfa de 0,05 y poder de $80 \%$. El análisis de los datos se realizó con el programa estadístico MedCalc, con aplicación de prueba de significancia t-student.

\section{Resultados}

Se reclutaron 124 pacientes, con edades entre 5 y 14 años, con un puntaje en el cuestionario CAN mayor o igual a 8, de los cuales 91 fueron contactados telefónicamente, e invitados al taller de educación. Finalmente asistieron 49 pacientes con sus respectivos cuidadores. Se realizó un total de 25 talleres, con un promedio de asistencia de 1,96 niños por taller, con un rango de asistencia de 1 a 4 niños por taller, y adicionalmente sus cuidadores. En la segunda etapa, asistieron 40 pacientes, lo que arrojó una pérdida de 18\% (9 pacientes).

El resultado de los 40 niños se describe a continuación: Previo al taller de educación, el puntaje CAN promedio fue 16 puntos y el puntaje CAN promedio, posterior al taller de educación, fue 7,7 puntos $(p=0,0001)$. En el 52,5\% $(n=21)$ de los pacientes, se logró puntaje CAN menor a 8 puntos después del taller de educación, indicador de buen control. Al analizar los tratamientos utilizados por los pacientes, antes y después de asistir al taller, se excluyeron 2 pacientes por modificación del tratamiento entre las evaluaciones. Al excluir estos dos pacientes, que modificaron tratamiento, el nuevo grupo considerado para análisis, fue de 38 pacientes, las características de este grupo se presenta en la Tabla 1.

Al realizar el análisis de los 38 pacientes, se obtuvo el siguiente resultado: el 47,5\% $(n=19)$ de los pacientes, disminuyó su puntaje CAN a menos de 8 puntos. La nota promedio de la prueba de conocimientos, previo a educación, fue 4,9, la que mejoró a 6,5 posterior al taller de educación $(\mathrm{p}=0,0001)$. Sobre la evaluación de la técnica inhalatoria, la nota promedio previo al taller, fue 4,3, la que aumentó a 6,1 post taller $(\mathrm{p}=0,0001)$. En relación a la adherencia (días sin tratamiento en la semana), antes del taller fue 1,21 días/semana, y post taller fue 0,42 días/ semana $(p=0,001)$. Al ser evaluado el conocimiento, que tenían los cuidadores sobre los nombres del inhalador que utilizaban los niños, antes del taller, 10 cuidadores no sabían el nombre del inhalador, y luego del taller sólo uno no era capaz de entregar esta información.

Al evaluar en forma diferencial, el grupo de pacientes que logró un puntaje CAN menor a 8

Tabla 1. Características generales de los grupos de niños asmáticos según puntaje del cuestionario de control de asma: CAN post taller de educación

\begin{tabular}{|c|c|c|c|}
\hline & Grupo total $(n=38)$ & $\begin{array}{l}\text { Grupo CAN }<8 \text { puntos } \\
(n=19)\end{array}$ & $\begin{array}{l}\text { Grupo CAN }>8 \text { puntos } \\
(n=19)\end{array}$ \\
\hline Edad promedio (rango) & $\begin{array}{l}7,5 \text { años } \\
\text { (5 a } 14 \text { años) }\end{array}$ & $\begin{array}{l}8,1 \text { años } \\
(5 \text { a } 14 \text { años })\end{array}$ & $\begin{array}{l}\text { 7,5 años } \\
(5 \text { a } 14 \text { años })\end{array}$ \\
\hline Sexo & $52 \%$ masculino & 10/19 masculino & 10/19 masculino \\
\hline Clasificación asma & $\begin{array}{l}\text { Asma severa } 13 / 38 \\
\text { Asma Moderada } 24 / 38 \\
\text { Asma leve } 1 / 38\end{array}$ & $\begin{array}{l}\text { Asma severa } 10 / 19 \\
\text { Asma moderada } 9 / 19 \\
\text { Asma leve } 0\end{array}$ & $\begin{array}{l}\text { Asma severa } 3 / 19 \\
\text { Asma moderada } 15 / 19 \\
\text { Asma Leve } 1 / 19\end{array}$ \\
\hline Tratamiento* & $\begin{array}{l}\text { CI } 7 / 38 \\
\text { CI+LABA } 31 / 38\end{array}$ & $\begin{array}{l}\text { CI 2/19 } \\
\text { CI+LABA 17/19 }\end{array}$ & $\begin{array}{l}\text { CI } 5 / 19 \\
\text { CI+LABA } 14 / 19\end{array}$ \\
\hline CAN Pre-educación & $16,23(8$ a 30$)$ & $14,6(8$ a 30$)$ & 17,8 (8 a 29) \\
\hline CAN Post-educación & 7,7 (1 a 22) & $3,8(1$ a 7$)$ & $11,5(8$ a 22$)$ \\
\hline
\end{tabular}

*CI: Corticoides inhalados. LABA: Beta agonistas de acción prolongada. 
Tabla 2. Evaluación del grupo de niños asmáticos con puntaje CAN menor a 8 post taller de educación $(n=19)$

\begin{tabular}{|lccc|}
\hline Variable & Pre taller & Post taller & p \\
Prueba conocimientos & $5,1($ IC 4,7-5,4)* & $6,5($ IC 6,2-6,7)* & 0,0001 \\
Técnica inhalatoria & $4,3($ IC 3,8-4,8)* & $6,3($ IC 6-6,6)* & 0,0001 \\
Adherencia a tratamiento & $1,47(0,69-2,2)^{\circ}$ & $0,47(0,17-0,76)^{\circ}$ & 0,02 \\
Conocimiento fármaco & $79 \%(15 / 19)$ & $100 \%(19 / 19)$ & \\
\hline
\end{tabular}

*Notas promedio. ${ }^{\circ}$ Promedio días/semana. IC: intervalo de Confianza.

Tabla 3. Evaluación del grupo de niños asmáticos con puntaje CAN mayor o igual $8(n=19)$ post taller de educación

\begin{tabular}{|lccc|}
\hline Variable & Pre taller & Post taller & p \\
Prueba conocimientos & $4,78($ IC 4-5,1)* & $6,52($ IC 6,3-6,7)* & 0,0001 \\
Técnica inhalatoria & $4,2($ IC 3,5-4,98)* & $5,8($ IC 3,3-6,3)* & 0,0001 \\
Adherencia a tratamiento & $0,94(0,5-1,3)^{\circ}$ & $0,36(0,08-0,6)^{\circ}$ & 0,0075 \\
Conocimiento fármaco & $68 \%(13 / 19)$ & $94 \%(18 / 19)$ & \\
\hline
\end{tabular}

*Notas promedio. ${ }^{\circ}$ Promedio días/semana. IC: intervalo de Confianza 95\%.

después del taller de educación $(\mathrm{n}=19)$ y el grupo de pacientes que no logró disminuir el puntaje a menos de 8 puntos $(n=19)$, ambos grupos presentaron resultados similares respecto a nota de conocimientos, nota en técnica inhalatoria y reducción de los días sin administrar el tratamiento en la semana (Tablas 2 y 3). Al realizar el análisis de regresión múltiple, no se encontró relación entre las variables evaluadas. No se registraron hospitalizaciones en los pacientes, en el período que medió entre ambas sesiones.

Sobre la prueba de conocimientos, cabe destacar que el $72 \%$ de los cuidadores, estaba de acuerdo con la afirmación que indicaba que los inhaladores causan adicción o dependencia en sus hijos, respuesta que disminuyó a $35 \%$ post taller de educación. Además antes del taller el $75 \%$ de los cuidadores reconocía el asma como una enfermedad crónica, lo que aumentó a $88 \%$ post taller. Un tercio de los cuidadores antes del taller, reconocían el asma y alergia como la misma enfermedad, lo que se reduce a $7 \%$ post taller.

\section{Discusión}

Posterior a una intervención de carácter educativo, nuestro trabajo demostró mejoría en la técnica inhalatoria, adherencia y conocimientos de los cuidadores sobre Asma Bronquial. Respecto a nuestro objetivo principal, que era reducir el puntaje CAN, obtuvimos un resultado mejor a lo esperado, al reducir el puntaje CAN a menos de
8 puntos, en el $47 \%$ de los pacientes. El grupo de pacientes, que logró reducir el puntaje CAN, así como el grupo que no lo logró, no tuvo diferencias en incrementar los conocimientos, adherencia y optimizar la técnica inhalatoria. Dado lo anterior, no podemos atribuir a la educación, como factor exclusivo, el mejor control de la enfermedad, ya que en ambos grupos, se realizó la misma intervención, con similares resultados en los objetivos secundarios, pero sin lograr en todos, el resultado final, que fue mejorar el control del asma bronquial. Una posible solución, para resolver el rol de la educación, en el control del asma, puede ser replicar este estudio, con un grupo control, el cual no se someta a esta intervención educativa. Reconocemos como limitación de nuestro estudio, la ausencia de este grupo control, pero dado que la educación es un pilar en el tratamiento de las enfermedades crónicas, nos pareció adecuado, en esta primera instancia, ofrecer a todos los pacientes la oportunidad de mejorar los conocimientos básicos sobre su patología.

Nuestro trabajo, demuestra la importancia de la educación, en incrementar los conocimientos, tanto de los pacientes y sus cuidadores, sobre la enfermedad que los afecta, permitiendo derribar mitos sobre el tratamiento. Además permitió mejorar la adherencia y técnica inhalatoria, dos objetivos que son buscados, en nuestra práctica clínica diaria.

Lamentablemente, no todos los cuidadores que fueron invitados, accedieron a asistir a nuestro taller de educación, debido a diversas causas, 
como falta de tiempo por trabajo y colegio, así como por problemas económicos para asistir en forma extra al hospital. Es por lo anterior, que recomendamos realizar la educación de los pacientes, el mismo día que asisten a control en el policlínico. La forma de integrar la educación, en nuestra práctica clínica, debe adecuarse según nuestros recursos y disponibilidad de personal, pero creemos que se debe buscar las instancias para implementar la educación en el manejo del asma bronquial.

Nuestros resultados, además de ser novedosos en nuestro medio, son alentadores y motivadores para seguir trabajando en educación en asma, con la realización de trabajos con metodología y objetivos diferentes, no evaluados en este trabajo (reducción de hospitalización, visita a urgencias, función pulmonar).

\section{Bibliografía}

1.- BROWER A F, BRAND P L. Asthma education and monitoring: what has been shown to work. Paediatr Respir Rev 2008; 9: 193-9.
2.- BAILEY E J, CATES C J, KRUSKE S G, MORRIS P $\mathrm{S}$, BROWN N, CHANG A B. Culture-specific programs for children and adults from minority groups who have asthma. Cochrane Database Syst Rev 2009; Jan 21: (1).

3.- COFFMAN J, CABANA M, HALPIN H A, YELIN E. Effects of Asthma Education on Children's use of Acute Care Services: A Meta-analysis. Pediatrics 2008; 121 : 575-86.

4.- LIU C, FEEKERY C. Can Asthma education Improve Clinical Outcomes? An Evaluation of a Pediatric Asthma Education Program. J Asthma 2001; 38: 269-78.

5.- CABANES L, NEIRA M A, Grupo de Asma y educación (SENP). Asma y Educación. Monografías 2008. Sociedad de Neumología Pediátrica. 2008; 6-101.

6.- PÉREZ-YARZA E G, BADIA X, BADIOLA C, COBOS N, GARDE J, IBERO M, et al on behalf of the CAN Investigator Group. Development and Validation of a Questionnaire to Assess Asthma Control in Pediatrics. Pediatr Pulmonol 2009; 44: 54-63.

7.- VILLA J R, COBOS N, PÉREZ-YARZA E G, GARDE J M, IBERO M, BADIOLA C, et al. Punto de corte que discrimina el nivel de control del asma en el cuestionario del "control del asma en niños" (CAN). An Pediatr (Barc) 2007; 66 (Supl 2): 76-7.
Correspondencia a:

Dra. María Teresita Henríquez Young

3 Oriente 1270 departamento 133-B,

Edificio Toscana, Viña del Mar.

E-mail: terenb@gmail.com 